\title{
Electrochemical Paper-based DNA Sensor for Human Papillomavirus Detection
}

\author{
Prinjaporn Teengam $^{1}$, Weena Siangproh ${ }^{2}$, Adisorn Tuantranont ${ }^{3}$, Charles S. Henry ${ }^{4}$, Tirayut Vilaivan ${ }^{5}$, \\ Orawon Chailapakul, \\ ${ }^{1}$ Program in Petrochemistry, Chulalongkorn University, Pathumwan, Bangkok, 10330, Thailand \\ ${ }^{2}$ Department of Chemistry, Faculty of Science, Srinakharinwirot University, Bangkok, 10110, Thailand \\ ${ }^{3}$ Nanoelectronics and MEMS Laboratory, National Electronics and Computer Technology, \\ Pathumthani, 12120, Thailand \\ ${ }^{4}$ Department of Chemistry, Colorado State University, Fort Collins, Colorado 80523, United State \\ ${ }^{5}$ Organic Synthesis Research Unit, Department of Chemistry, Faculty of Science, Chulalongkorn \\ University, Pathumwan, Bangkok, 10330, Thailand \\ ${ }^{6}$ Electrochemistry and Optical Spectroscopy Center of Excellence, Department of Chemistry, \\ Chulalongkorn University, Pathumwan, Bangkok, 10330, Thailand \\ ${ }^{7}$ National Center of Excellence for Petroleum, Petrochemicals, and Advanced Materials, \\ Chulalongkorn University, Pathumwan, Bangkok, 10330, Thailand
}

\begin{abstract}
A novel paper-based electrochemical biosensor using an anthraquinone-labeled pyrrolidinyl peptide nucleic acid (acpcPNA) probe (AQ-PNA) and graphene-polyaniline (G-PANI) modified electrode was developed for human papillomavirus (HPV) detection. An inkjet printing technique was employed to prepare the paper-based G-PANI-modified working electrode. The AQ-PNA probe baring a negatively charged amino acid at the $\mathrm{N}$-terminus was immobilized onto the electrode surface through electrostatic attraction. Electrochemical impedance spectroscopy (EIS) was used to verify the AQ-PNA immobilization. The paper-based electrochemical DNA biosensor was used to detect an oligonucleotide target with a sequence corresponding to HPV type 16 by measuring the electrochemical signal response of the $A Q$ label using square-wave voltammetry before and after hybridization. After the addition of target DNA, the current signal significantly decreased. This phenomenon is explained by the rigidity of PNA-DNA duplexes, which obstructs the accessibility of electron transfer from the $A Q$ label to the electrode surface. Under optimal conditions, the linearity in the range of 10-200 $\mathrm{nM}$ with the detection limit of $2.3 \mathrm{nM}$ was obtained. The performance of this biosensor on real DNA samples was tested with the detection of PCR-amplified DNA samples from the $\mathrm{SiHa}$ cell line. The proposed method employs an inexpensive and disposable device and is promising for the screening and monitoring of the amount of HPV-DNA type 16 to identify the primary stages of cervical cancer.
\end{abstract}

Key words: Paper-based DNA sensor, Human Papillomavirus, Graphene, Polyaniline, acpcPNA 